\title{
RESEARCH ON LANE DETECTION TECHNOLOGY BASED ON OPENCV
}

$$
\text { Xu Yang }{ }^{1, ~ a ~} \text {, Zhang Ling }{ }^{2, b}
$$

${ }^{1}$ School ofChongqing University of Posts and Telecommunications, Chongqing 400065, China;

${ }^{2}$ School of Chongqing University of Posts and Telecommunications, Chongqing 400065, China;

axuyang@cqupt.edu.cn, b18996105054@163.com

Keywords: OpenCV, Image processing, LMedSquare, Lane detection.

\begin{abstract}
In view of the huge computing, poor anti-interference ability of traditional detection alogrithm, it does not meet the requirement of the vehicle system, for which this paper proposed a lane detection method based on OpenCV. Preprocessing image in the OpenCV environment, adopting LMedSquare(Least Median Square) idea to select the best subset combined with least squares method to picewise fitting the lane so that it realized automatic identification of lane. This algorithm is suitable for both straight and curve. Simulation shows that this algorithm has well real-time performance, accuracy and robustness. It can meet the requirements of the vehicle system.
\end{abstract}

\section{Introduction}

As the core content of vehicle collision warning system, the lane line detection and recognition widely used in automotive systems.Its accuracy, real-time and robustness are directly related to the safety of the car and the driver.The current research adopts different detection algorithm for straight and curve. This increased the complexity of the algorithm.Hough transform is widely used in the lane line detection, but its computational complexity and real-time performance don't meet the requirements of vehicle system.Least-square method is also commonly used in lane detection, but its sensitivity to noise and poor anti-interference abilityaffected the accuracy of lane line identification[1].

To solve problemsabove, this paper put forward an idea which based on LMedSquare to select the best subset, combined with the least square method to piecewise fitting algorithm. This method can eliminate the unnecessary noise in the search process for the lane line feature points so that improving the system's anti-noise ability. The algorithm applies to both straight and curve and reduces the complexity of the algorithm. At the same time,it meets the real-time requirement of the vehicle system.

\section{OpenCV Overview}

OpenCV is an open source computer vision library. The library is written in $\mathrm{C}$ and $\mathrm{C}++$ and runs under Linux, which provides great convenience for image processing.Its powerful image and matrix operation ability shorten the development cycle for the developers[2]. The OpenCVlibrary contains over 500 functions that span many areas invision including factory product inspection, medicalimaging, security, and user inter face, camera calibration,stereo vision and robotics.

\section{The Description and Implementation of Lane Line Identification Algorithm}

The flow chart of main program is shown as Fig.1.

There are about 4 steps of this algorithm, I) Captureand input video signal II) preprocessing on thevideosignals, including gray processing, denoising, image binarization III) select the best subset based onLMedSquare, thenget the lane line of the image using the least square method.IV) Output the detective result. 


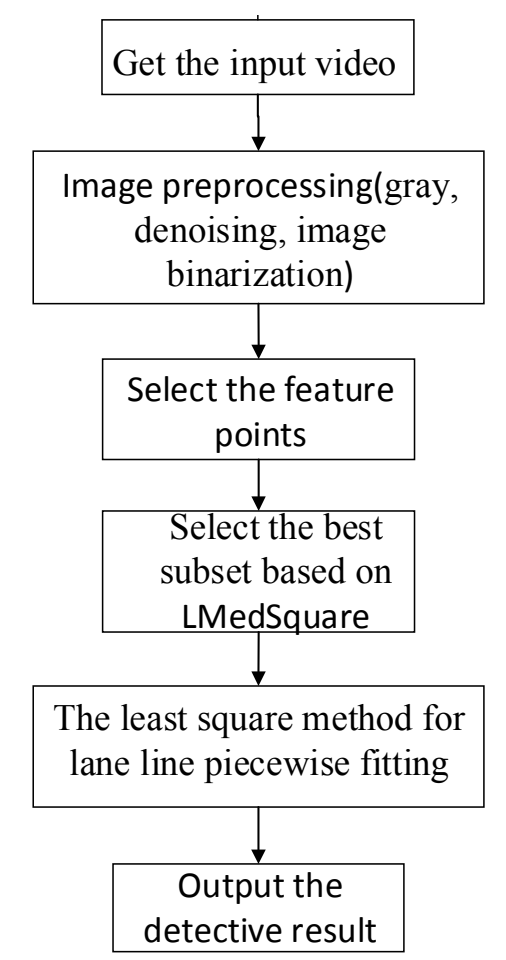

Fig. 1flow chart of main program

\subsection{Capture and Input Video Signal}

The algorithm adopts a camera as an input device. You can initializethe camera by using the cvCamInit() function. Then, itobtains the video streams as the system's input datathrough the OpenCV library function cvCreateCameraCapture().OpenCVcan also divide video filesinto frames.At last, we utilized algorithm inthis paper to deal with the frames.

\subsection{Preprocess the Input Video Signals.}

Graying. The video acquired through the camera is RGB color images. The computational cost is too largeif handled it directly.In this paper, in order to remove redundant information andimprove the processing speed,we usedthe weighted average method to transform thecolor video to a grayscale video.In OpenCV function library,Image gray scale can realize through the cvCvtColor(constCvArr* src, CvArr* dst,int code)function.

Image Denoising.Thevideo signalwe acquiredcontains a lot of noise which affects the accuracy of image recognition. Therefore, we need to filter the image.Filtering include spatial domain filtering and frequency domain filtering. Frequency domain filtering need two domain transform which increased image computation. In this paper, we adopted the spatial domain filtering method to process the noisy image.

Binaryzation. There is still useless information after the image denoising.In order to improve the system's real-time performance,we must carry on the binarization processing to distinguish the target object and the background.we got the binary image by using the Ostu threshold method. The advantage of Ostu threshold method is selecting thresholdautomatically, it does not need to set the parameters by artificially.

As shown in Fig.2, (a) shows the original image(b)shows the grayscale image (c) shows the denoised image(d) shows the binary image got by the Ostu threshold method.
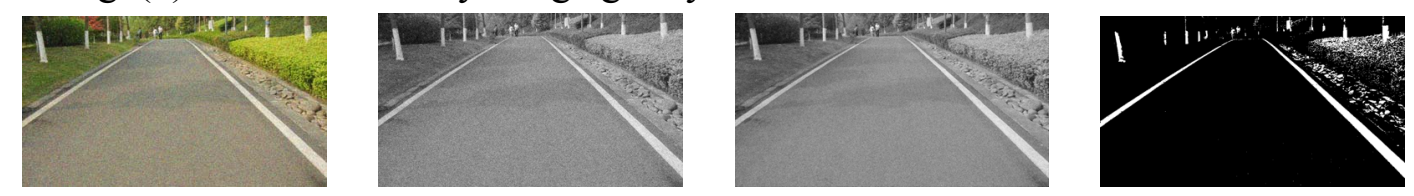

(a) original image(b) grayscale image(c) denoised image(d) binary image

Fig. 2 The example of preprocessing on input image 


\subsection{Select the Best Subset Based On LMed Square}

The large computation of Hough transform cannot satisfy the system real-time requirement.The least squares curve fitting is sensitive to noise.In this paper, we use LMedSquareideato select the best subset combined with the least square method for lane line piecewise fitting.

The implementation process of LMedSquare as follow:

Assuming that the proportion of noise points in all feature pointsise, each feature subset contains $\mathrm{s}$ feature points. Then, to reach $95 \%$ credibility the minimum number of sample subset $m_{s}$ is [3]:

$m_{s}=\frac{\log (1-p)}{\log \left(1-(1-\varepsilon)^{\varepsilon}\right)}(1)$

Randomly selected feature points assembled feature subset,assuming that feature subset contains $\mathrm{n}$ feature points, the curve parameter vector is $\vec{P}$, and the number of parameters is $p$. Each subset has a parameter vector initial value and repeats the following process:

1) Initialize the parameter vector $\overrightarrow{p_{j}}[4]$

2) Calculate the redundancy $d_{i}$ of each point for the given parameter vector $\overrightarrow{p_{j}}$;

3)Calculate the median of redundancy square $m_{j}$. And get the minimum $d_{i}$ named $M_{J}$ :

$M_{J}=\min _{j=1,2 \ldots m_{s}} \operatorname{med}_{i=1,2 \ldots n} d_{i}^{2}$

Redundancy standard deviation was initial estimated to be $\sigma_{0}$ :

$\sigma_{0}=1.4826\left(1+\frac{5}{\mathrm{n}-\mathrm{p}}\right) \sqrt{M_{J}}$

Using $\sigma_{0}$ give a weight coefficient $\omega_{i}$ for each feature point[5]:

$\omega_{i}=\left\{\begin{array}{l}1, d_{i}^{2} \leq\left(2.5 \sigma_{0}\right)^{2} \\ 0, \text { otherwise }\end{array}\right.$

Standard deviation was final estimated to be $\sigma_{0}{ }^{*}$ :

$\sigma_{0}^{*}=\sqrt{\sum_{i=1}^{n}\left(\omega_{i} d_{i}^{2}\right) /\left(\sum_{i=1}^{n} \omega_{i}-p\right)}$

If $d_{i}$ is over $k$ times $(k=1.96)$, we can seem it as noise pointand exclude it.In the best subset constitute of the remaining data,using the least square method process piecewise fitting curve and will get more accurate edge curve.

\section{Result of Experiment}

In order to verify the validity and the robustness of the lane line algorithm,we make a large number of experiments.Using OpenCV function cvCreateCameraCapture() to divide video filesinto frames.Respectively use tradition Hough transformation,improved Hough transformation,least square method and the detection algorithm in this paper to make detection test. The comparison of the identification result shown in table 1.

Table 1 Contrasts of the performance of the lane line detection algorithm

\begin{tabular}{lccccc}
$\begin{array}{r}\text { performance } \\
\text { parameter }\end{array}$ & $\begin{array}{c}\text { run } \\
\text { time/s }\end{array}$ & $\begin{array}{c}\text { Horizontal } \\
\text { Error/cm }\end{array}$ & $\begin{array}{c}\text { Accuracy } \\
\text { rate }\end{array}$ & $\begin{array}{c}\text { false } \\
\text { alarm } \\
\text { rate }\end{array}$ & $\begin{array}{c}\text { missing } \\
\text { alarm } \\
\text { rate }\end{array}$ \\
algorithm & 163 & 5.7 & $47.4 \%$ & $30.0 \%$ & $52.4 \%$ \\
Tradition Hough & 89 & 8.2 & $28.7 \%$ & $28.7 \%$ & $47.3 \%$ \\
Improved Hough & 45 & 12.8 & $84.5 \%$ & $12.0 \%$ & $15.5 \%$ \\
Least square method & 68 & 7.5 & $97.2 \%$ & $3.1 \%$ & $2.8 \%$ \\
Algorithm in this paper & 68 & & & & \\
\hline
\end{tabular}

We can know from the comparison result in table 1,tradition Hough transformation and improved Hough transformationhavelong running time and poor accuracyrate for the curve.Affected by the noise, thehorizontal errorof least squares method for lane line detection is too large.Using the detection algorithm in this paper,the detection time of the single framehas been controlled within $68 \mathrm{~ms}$, it satisfy the system's real-time requirement.In the experiment for 1000 frames, the 
identificationaccuracy rate reached $97.2 \%$. At the same time, it has a very low false alarm rate and missing alarm rate.There are some examples on different conditionsShown as Fig.6.

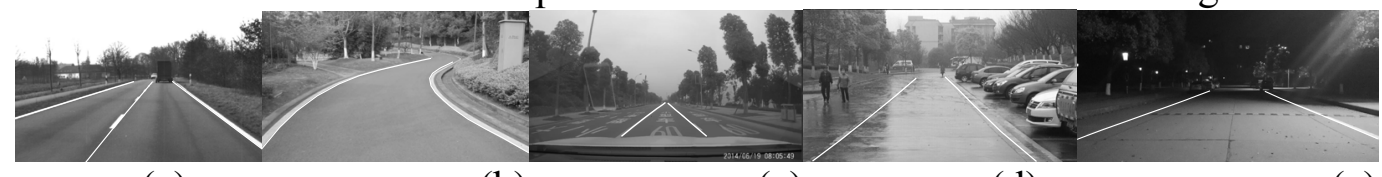

(a)

(b)

(c)

(d)

(e)

Fig. 6 Result of different conditions, (a)multilane (b)curve (c)identify interference(d)rainy days and the lane line is damaged (e)night

\section{Summary}

A research onthe lane line detection based on OpenCVis presented in this paper.Using LMedSquare idea to select optimum subset in the lane line feature points so that eliminating unnecessary noise and improving the system's anti-noise ability.Then, a least squares method piecewise fit to the lane line in the optimum subset.This algorithm applies to both straightway and curve witha good detection effect and high recognition rate. The algorithm is transplanted into the embedded platform to provide accurate and effective information for the vehicle. So ithas significant application value.

\section{Acknowledgments}

This paper is project supported by Natural Science Foundation Project of CQ CSTC cstc2012jjA60002.

\section{Reference}

[1]. YOO H, YANG U, SOHN K. Gradient-enhancing conversion for illumination-robust lane detection. 14(3) 1083-1094.

[2]. GAIKWAD V, LOKHANDE S. Lane departure identification for advanced driver assistance. 16(2) 910-918.

[3]. AharonBar Hillel, Ronen Lerner, Dan Levi, et al. Recent progress in road and lane detection: A survey. 25 727-745.

[4]. GOPALAN R, TSAI H, SHNEIER M, et al. A learning approach towards detection and tracking of lane markings. 13(3) 1088-1098.

[5]. CUALAIN D O, GLAVIN M, JONES E. Multiple camera lane departure warning system for the automotive environment. 6 (3) 223-234. 